\title{
Research of an improved routing protocol in wireless network
}

\author{
Shunye Wang \\ Langfang Teachers University, Langfang, 065000, China
}

\begin{abstract}
Keywords: MANET; AODV; DSR; DSDV; Network Simulation-2.
\end{abstract}
\begin{abstract}
The field of MANET has gained an important part of the interest of researchers and become very popular in last few years. MANET can operate without fixed infrastructure and can survive rapid changes in the network topology. The main method for evaluating the performance of MANET is simulation. This paper is subjected to the on-demand routing protocols with identical environment conditions and evaluates their relative performance with respect to the four performance metrics. We investigated various simulation scenes with different moving speed. From the detailed simulation result and analysis, a suitable routing protocol can be chosen for a specified network and goal.
\end{abstract}

\section{Introduction}

Mobile Ad hoc Network (MANET) is the most innovative and challenging area of wireless networking. It is a collection of mobile devices and is self-configuring, dynamic topologies, multi hop wireless network which forms a communication network via multi hop wireless network connection without any central control. Nodes in the network communication with another only if it lies within its transmission range. MANET has potential applications in civilian and military environments [1].

Routing protocol which is aimed to forward data is an important part not only wired network but also wireless network. At the same time the routing protocols used in ordinary wired networks are not well suited for this kind of dynamic environment. Routing algorithms are often difficult to be formalized into mathematics they are instead tested using extensive simulation. The main protocols identify to two classifications, proactive protocol and on-demand routing protocol. In proactive routing protocols, each node maintains routing information to every other node in the network. The routing information is usually kept in a number of different tables. These tables are periodically updated and/or if the network topology changes. On-demand routing protocols build and maintain only needed routes to reduce routing overheads. In on-demand protocols, a route discovery process is initiated whenever a route is needed. Each node in on demand routing does not need periodic route table update exchange and does not have a full topological view of the network. Network hosts maintain route table entries only to destinations that they communicate with [2].

The structure of the rest of the paper is as follows. Section II discusses the each routing protocols such as DSDV, DSR and AODV. Section III describes the performance parameters in the experimental, the simulation scene setting and the experiment analysis. And finally, Section IV discusses about the conclusion and future works.

\section{Routing protocols}

\subsection{Destination-sequenced distance vector (DSDV).}

The DSDV is a proactive protocol. It provides a single path to a destination, which is selected using the distance vector shortest path routing algorithm. In order to reduce the amount of overhead transmitted through the network, two types of update packets are used. These are referred to as a "full dump" and "incremental" packets. The full dump packet carries all the available routing information and the incremental packet carries only the information changed since the last full dump. The incremental update messages are sent more frequently than the full dump packets. However, DSDV still introduces large amounts of overhead to the network due to the requirement of the periodic 
update messages. So the protocol is not scale in large network since a large portion of the network bandwidth is used in the updating procedures [3].

\subsection{Dynamic source routing (DSR).}

As stated earlier, the DSR protocol requires each packet to carry the full address (every hop in the route), from source to the destination. This means that the protocol will not be very effective in large networks, as the amount of overhead carried in the packet will continue to increase as the network diameter increases. Therefore in highly dynamic and large networks the overhead may consume most of the bandwidth. However, this protocol has a number of advantages. In small to moderately size networks (perhaps up to a few hundred nodes), this protocol may perform better. An advantage of DSR is that nodes can store multiple routes in their route cache, which means that the source node can check its route cache for a valid route before initiating route discovery, and if a valid route is found there is no need for route discovery. This is very beneficial in network with low mobility. Since they routes stored in the route cache will be valid longer. Another advantage of DSR is that it does not require any periodic beaconing, therefore nodes can enter sleep node to conserve their power. This also saves a considerable amount of bandwidth in the network [3].

\subsection{Ad hoc on-demand distance vector (AODV).}

The AODV enables dynamic, self-initiating, multi-hop routing between participation mobile nodes wishing to establish and maintain an MANET and other wireless ad hoc networks [4]. AODV is an on-demand routing protocol.

The operation of the protocol has two phases: route discovery and route maintenance. In AODV, when a route is needed to some destination, the protocol start route discovery. It broadcasts a RREQ for the destination to its neighbours. A node on receiving the RREQ checks if it has received the same request before using the Route-ID. It is not the destination, it broadcasts the RREQ and at the same time backward route to the source is created [5]. If RREQ receiving node is destination, it creates RREP. The RREP reached at source node hop by hop. When RREP broadcasts, each intermediate node establishes a route to the destination. When RREP is received by source, it stores the forward route to the destination and starts sending data. If the source receives multiple RREPs, the route selects the route with less number of hops [6].

\section{Simulation and analysis}

\subsection{Performance Indices.}

Packet Delivery Fraction (PDF) [2]: The ratio of the data packets delivered to the destinations to those generated by the sources. Mathematically, it can be expressed as:

$$
P=\frac{1}{C} \sum_{f=1}^{C} \frac{R_{f}}{S_{f}}
$$

Where $P$ is the fraction of successfully delivered packets, $C$ is the total number of flow or connections, $f$ is the unique flow id serving as index, $R_{f}$ is the count of packets received from flow $\mathrm{f}$ and $S_{f}$ is the count of packets transmitted to $f$.

Average end-to-end delay [2]: This includes all possible delays caused by buffering during route discovery latency, queuing at the interface queue, retransmission delays at the MAC, and propagation and transfer times. It can be defined as:

$$
D=\frac{1}{N} \sum_{i=1}^{s}\left(R_{i}-S_{i}\right)
$$

Where $N$ is the number of successfully received packets, $i$ is unique packet identifier, $S$ is the total number of packets which is received, $R_{i}$ is time at which a packet with unique id i is received, $S_{i}$ is time at which a packet with unique id $i$ is sent and $D$ is measured in ms.

Normalized routing load: The number means routing control packets that are needed to send an effective data packets. It reflects the situation of the network congestion in the transmission procedure and the efficiency of power supply of node. The routing protocol which has bigger value of routing load may have more possible of congestion. It can be define as: 


$$
L=\left(S_{n}+F_{n}\right) / R_{n}
$$

Where $L$ is the normalized routing load, $S_{n}$ is the routing packets that is sent, $F_{n}$ is the retransmission routing packets, $R_{n}$ is the number of valid data packets that is received.

Average end-to-end throughout: It measures all the packets that received per unit of time. The packets not only include valid data packets but also include the extra packets in order to routing and forwarding.

$$
T=\left(M_{t}+N_{t}\right) / t
$$

Where $T$ is the average end-to-end throughout, $M_{t}$ is sum of CBRs and the $N_{t}$ is the extra packets in order to routing and forwarding, $t$ is total time it takes, and $T$ is measured in $\mathrm{b} / \mathrm{s}$.

\subsection{Simulation scene setting.}

The simulations were performed using Network Simulator 2 (NS-2) [7]. The mobility model uses 'random waypoint model' in a rectangular filed of $800 \mathrm{~m}$ x $800 \mathrm{~m}$ with 50 nodes. The mobile scene file differently use by different mobile. During the simulation, each node starts its journey from a random spot to a random chosen destination with a speed. Once the destination is reached, the node takes a rest period of time in second mobile with another random speed and another random destination is chosen. In the whole process of simulation, the node will always repeat the above process. The entire simulation time is 100 s.

In the simulation, it defines 6 different mobile speed mode, the definition of mobile maximum speed are respectively $1 \mathrm{~m} / \mathrm{s}, 10 \mathrm{~m} / \mathrm{s}, 20 \mathrm{~m} / \mathrm{s}, 30 \mathrm{~m} / \mathrm{s}, 40 \mathrm{~m} / \mathrm{s}, 50 \mathrm{~m} / \mathrm{s}$. The pause time is 0 , which means it is a continuous movement. The traffic sources are CBR (Constant Bit Rate) flowing from source to destination. To ensure the experimental results consistency, it uses the same data scene files in all the experiments, the scene in the simulation has 50 nodes, the maximum number of connection is 40 , and it sends 4 packets in per second.

\subsection{Simulation and analysis.}

The simulation is based on the scene ahead, and the measure is based on performance metric.

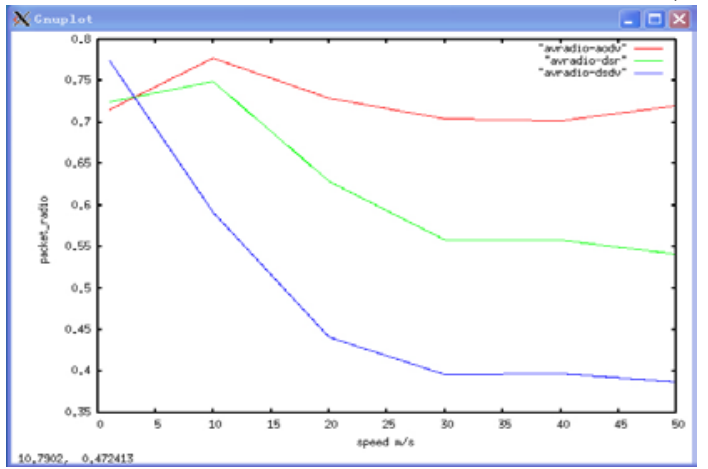

Fig.1 Packet delivery fraction

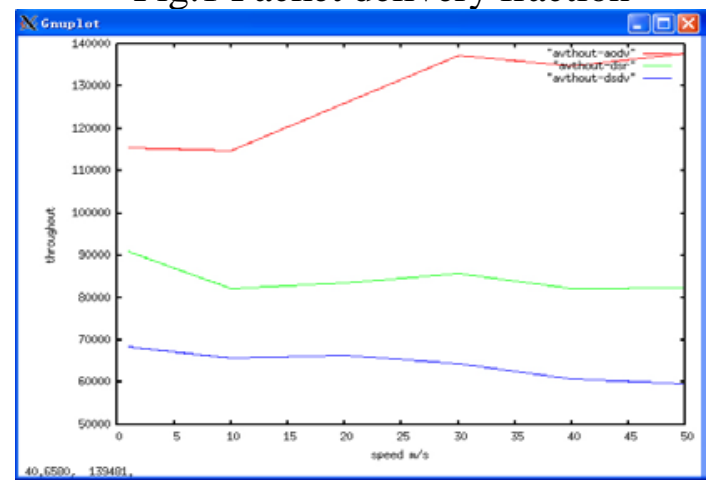

Fig. 4 Average end-to-end throughout

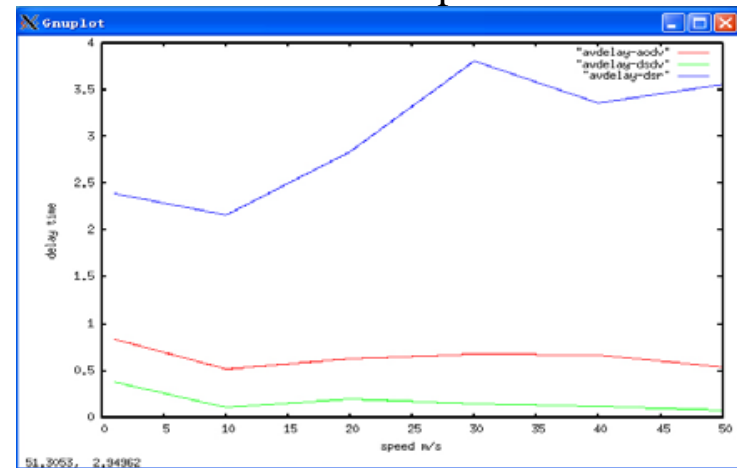

Fig.2 Average end-to-end delay

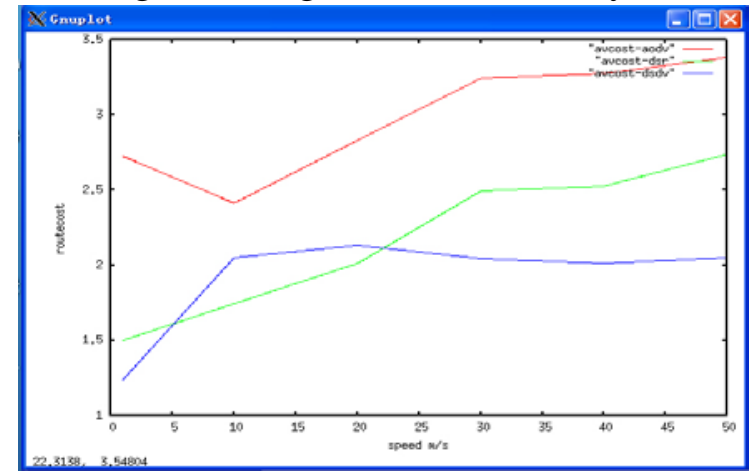

Fig.3 Normalized routing load

From the Fig. 1 about the packet delivery fraction: With the increase of node moving speed, the packet delivery fractions all are on decline. The DSDV decreases greatly and abruptly to a very small value. DSR also decreases greatly, but in the whole experiment, it always greater than the DSDV. At slow speed, the values of all the three protocols get more than $70 \%$. At the speed of $50 \mathrm{~m} / \mathrm{s}$, the AODV is still about $70 \%$, while DSDR is not up to $40 \%$ and DSR is only about $55 \%$. 
From the Fig. 2 about the average end-to-end delay, with the increase of the maximum moving speed, the time of delay about AODV, DSR and DSDV are all increase. The effect of moving speed on the DSDV is more than the other two protocols which are relatively stable. And the time of DSR is longer greatly AODV and DSDV.

From the Fig. 3 about the average end-to-end throughout, the value increase about the AODV with the increase of moving speed while the DSR and DSDV decrease. This is because AODV is an on-demand routing protocol, the bandwidth increase unceasingly in route discovery at higher speed. And to transmit valid data packet there need more routing forward packets.

From the Fig. 4 about the normalized routing load, they all increase with the higher moving speed. This is true in real situation, the movement is faster, the network topology changes greater, it also means there should more data packet to maintain and update the routing table. As shown in the diagram, the routing load of DSR is less than AODV. It is because the DSR uses the caching and promiscuous pattern to receive the route request packets.

Through the simulations in six different moving speeds, we can find that the AODV routing protocol has better performance than DSR and DSDV at fast moving speed. At the same time, to get superior performance, AODV needs to rely on higher routing load and consume more bandwidth to maintain. When the moving speed is very slow DSDV protocol has higher availability, because the routing overhead and bandwidth consumption is lower.

\section{Summary}

As a special type of network, MANET has received increasing research attention in recent years. There are many active research projects concerned with MANET. MANET has applications in rapidly deployed and dynamic military and civilian systems. This work is an attempt towards a comprehensive performance evaluation of three commonly used in MANET (DSDV, DSR and AODV). In this paper, using the latest simulation environment NS-2, we evaluated the performance of three widely used routing protocols using packet-level simulation in different moving speed. In short, AODV has the best all round performance. DSR is suitable for networks with moderate mobility rate. It has low overhead that makes it suitable for low bandwidth and low power network. DSDR is suitable for operation in low moving speed mobile networks.

\section{Acknowledgments}

This work was supported in part by the Natural Science Foundation of LangFang Teachers College in 2013(LSZY201306). I also thank to my family especially my little son.

\section{References}

[1] Shunye Wang, Huiying Tian, Journal of Chemical and Pharmaceutical Research, 2014, 6(6), pp. 2168-2172.

[2] Anuj K. Gupta, Member, IACSIT, Dr. Harsh Sadawarti, Dr. Anil K. Verma, International Journal of Engineering and Technology, Vol.2, No.2, April 2010, pp. 226-231.

[3] Mehran Abolhasan. Tadeusz Wysocki. Eryk Dutkiewicz, Ad Hoc Networks, Vol. 2, No. 1, January 2004, pp. 1-22.

[4] K.Vanaja, Dr. R.Umarani, International Journal of Electronics and Computer Science Engineering, 2012 (9), pp. 1080-1086.

[5] ChiragBhalodia, Prof AmitM.Lathigara, Internatinal Journal of Advance Engineer and Research Development, 2014 (5), pp.1-9.

[6] Jooti Jain, Roopan Gupta, I.K.Bandhopadhyay, International Journal of Computer Application, 2011 (5), pp. 20-25.

[7] NS-2 Network simulator http://www.isi.edu/nsnam/ns. 